\title{
Long-term computed tomographic changes in cystic fibrosis patients treated with ivacaftor
}

\begin{abstract}
To the Editor:
Cystic fibrosis (CF) patients with gating mutations in the gene coding for the CF transmembrane conductance regulator (CFTR) protein have been the first to benefit from CFTR-targeted therapies since the approval of ivacaftor in 2012 by the Food and Drug Administration and the European Medicines Agency. Ivacaftor is a potentiator that increases chloride transport through the defective CFTR protein $[1,2]$. In phase III clinical trials, ivacaftor significantly improved lung function and clinical status in patients with a G551D mutation aged $\geqslant 6$ years $[3,4]$. In the two studies conducted in adults and children, the forced expiratory volume in $1 \mathrm{~s}(\mathrm{FEV} 1)$ showed a $10.4 \%$ predicted and $12.5 \%$ pred point increase from baseline after 24 weeks of treatment, as compared to a 0.1 and $0.2 \%$ pred point decrease in the control group. Clinical efficacy of ivacaftor was also shown in patients with other gating mutations of the CFTR gene, with a 7.5\% pred point increase in FEV1 after 8 weeks of treatment [5]. In all these phase III studies, the primary endpoint was the change in FEV1 and secondary endpoints were other clinical and functional parameters, such as change in weight, respiratory exacerbation rate, health status, and sweat-chloride concentration. Imaging was never listed as an endpoint. Data on computed tomography (CT) changes upon ivacaftor treatment are scarce [6-8] and long-term changes remain unknown. The aim of this study was to assess short- and long-term CT changes in adult CF patients treated with ivacaftor.
\end{abstract}

The study was approved by the Paris Ile de France I ethics committee. We conducted a multicentre retrospective study in eight French CF centres. Adult CF patients with at least one gating mutation and being treated with ivacaftor were included if they had a pre-therapeutic chest CT and at least one follow-up chest CT. Chest CT examinations were deemed eligible if they consisted in whole lung volumetric inspiratory CT acquisitions and if spirometric measurements were available within 1 month of CT examination. Chest CT examinations were performed on multidetector $\mathrm{CT}$ scanners, with acquisition parameters specific to each centre. CT images were reconstructed with thicknesses ranging from 0.6 to $4 \mathrm{~mm}$ and were visualised using the same pulmonary window settings (window width 1500, window level -450). A modified CF-CT scoring system was used, not including quantification of air trapping, since expiratory CT acquisitions were not available [9]. CT scoring was performed by one radiologist who had received 1-week intensive training in a reference centre (Lung Analysis, Erasmus Medical Center, Rotterdam, the Netherlands), in order to reach good scoring repeatability. Readings were performed in random order and the reader was blinded to patient identification, time of CT examination, results of previous CT readings and clinical data. 25 randomly selected CT scans were scored twice, with a 4-week interval, to verify intraobserver repeatability. Spirometry was performed in accordance with American Toracic Society/European Respiratory Society consensus guidelines [10]. FEV1 values expressed in percentage of predicted values were retrieved from the patient files.

Decision to perform CT as part of routine follow-up was left to the physician in charge of the patient, thus the frequency of CT examinations was not standardised. Examinations performed 3-18 months after the start of ivacaftor and those performed after 18 months of treatment were considered as short- and long-term follow-up CT examinations, respectively. FEV1 and CT scores at baseline (T0), short (T1) and long-term (T2) follow-up were compared using Wilcoxon test. Correlations between CF-CT score and FEV 1 , and between changes in CF-CT score and changes in FEV1 between two successive CT scans (inter-scan changes) were assessed using Spearman's correlation coefficients. Intraobserver repeatability was assessed using intraclass correlation coefficient (ICC). All analyses were performed with statistical software package "R" (version 3.2.1, R Foundation, Vienna, Austria).

22 adult patients (17 males and five females, mean \pm SD $36 \pm 11$ years) treated with ivacaftor were included. A total of $52 \mathrm{CT}$ examinations were available (22 at baseline and 30 during follow-up), and were scored with high intraobserver repeatability (ICC $0.88-0.96$ ). Eight patients had both short- and long-term follow-up CT examinations. Results are summarised in table 1 . At short-term follow-up, patients experienced a statistically significant FEV1 increase. Over the same period, CF-CT score, as well as two of its components, peribronchial thickening and mucus plugging, had significantly decreased, indicating improvement in CT scan abnormalities. At long-term follow-up and as compared to baseline, FEV1, CF-CT score, peribronchial thickening score and mucus plugging score had also significantly improved. In contrast, there was a significant increase of the bronchiectasis score (figure 1). Comparison between short- and long-term follow-up did not show any significant difference in FEV1 or CT-scores. There was a good correlation 


\begin{tabular}{|c|c|c|c|c|c|c|c|c|c|}
\hline & Baseline СТ (то) & $\begin{array}{l}\text { Short-term } \\
\text { follow-up (T1) }\end{array}$ & $\begin{array}{l}\text { Long-term } \\
\text { follow-up (T2) }\end{array}$ & $\begin{array}{l}\text { Pseudomedian } \\
\text { (95\% Cl) }\end{array}$ & $\mathrm{p}$-value & $\begin{array}{l}\text { Pseudomedian } \\
\text { (95\% Cl) }\end{array}$ & $\mathrm{p}$-value & $\begin{array}{l}\text { Pseudomedian } \\
\text { (95\% CI) }\end{array}$ & p-value \\
\hline Patients $\mathrm{n}$ & 22 & 14 & 16 & 14 & & 16 & & 8 & \\
\hline $\begin{array}{l}\text { Time from introduction } \\
\text { of ivacaftor months }\end{array}$ & $-4.1(-12.4-0.8)$ & $8(6.1-11.7)$ & $33.1(30.8-36.7)$ & & & & & & \\
\hline Bronchiectasis & $33.7(13.4-51.3)$ & $34.5(23.6-55.7)$ & $39.9(18.2-52.6)$ & $4.6(-0.9-9.5)$ & 0.133 & $5.0(1.5-8.1)$ & 0.025 & $2.3(-2.0-7.3)$ & 0.313 \\
\hline $\begin{array}{c}\text { Peribronchial } \\
\text { thickening }\end{array}$ & $43.5(36.3-55.4)$ & $43.5(27.0-48.1)$ & $40.8(27.8-48.1)$ & $-9.3(-17.6--0.90)$ & 0.042 & $-5.8(-17.3--3.1)$ & 0.002 & $-1.1(-8.3-6.6)$ & 0.833 \\
\hline Mucus plugging & $43.1(24.3-56.7)$ & $29.2(19.4-35.4)$ & $23.6(12.5-29.2)$ & $-18.1(-30.5--8.4)$ & 0.001 & $-19.4(-32.0--4.2)$ & 0.011 & $-7.3(-16.6-9.2)$ & 0.313 \\
\hline $\begin{array}{l}\text { Parenchymal } \\
\text { abnormalities }\end{array}$ & $7.4(3.9-10.7)$ & $8.2(3.7-10.7)$ & $7.1(3.3-7.4)$ & $-1.9(-3.7-0.4)$ & 0.083 & $-1.8(-2.8-0.2)$ & 0.119 & $1.7(-1.8-3.7)$ & 0.787 \\
\hline
\end{tabular}

Data are presented as median (interquartile range), unless otherwise stated. Comparison is assessed using paired Wilcoxon test and results.

\#: percentage of maximum possible scores.

between CF-CT score and FEV1 $(\mathrm{r}=-0.73, \mathrm{p}<0.001)$ and a moderate correlation between interscan changes of FEV1 and CF-CT scores $(\mathrm{r}=-0.51, \mathrm{p}<0.001)$.

To the best of our knowledge, this study is the first to report long-term CT changes in CF patients treated with ivacaftor. It is also the largest study reporting CT changes associated with ivacaftor and the first to evaluate its effect by using the CF-CT score on the whole lung following volumetric chest CT examination. Our results show a significant improvement of CF-CT scores on both short- and long-term follow-up. This improvement is mainly related to a significant decrease of the peribronchial thickening and mucus plugging scores, which are known to represent reversible CT changes during exacerbations in CF patients [11]. SHEIK et al. [8] reported similar improvement of peribronchial thickening, mucus plugging and Brody scores at 1 year follow-up in 10 patients treated with ivacaftor. However, they also found a significant decrease of the bronchiectasis score whereas we observed a significant increase of this score on long-term follow-up. This could be due to better visualisation after decrease of bronchial thickening and mucus plugging. Bronchiectasis results in irreversible changes with the destruction of elastic tissues [12]. The decrease in the bronchiectasis score reported in the study by SнEIK et al. [8] might be due to under-sampling, since scoring was based on only four CT images and not on the whole lung CT acquisition. Interestingly, we found most of the CT lesion improvement occurred during the first year of treatment and remained stable on long-term follow-up, a pattern for improvement that was also reported for lung function [13]. The good correlations we report between CT scores and FEV1 suggest CT is a valuable method for monitoring changes on
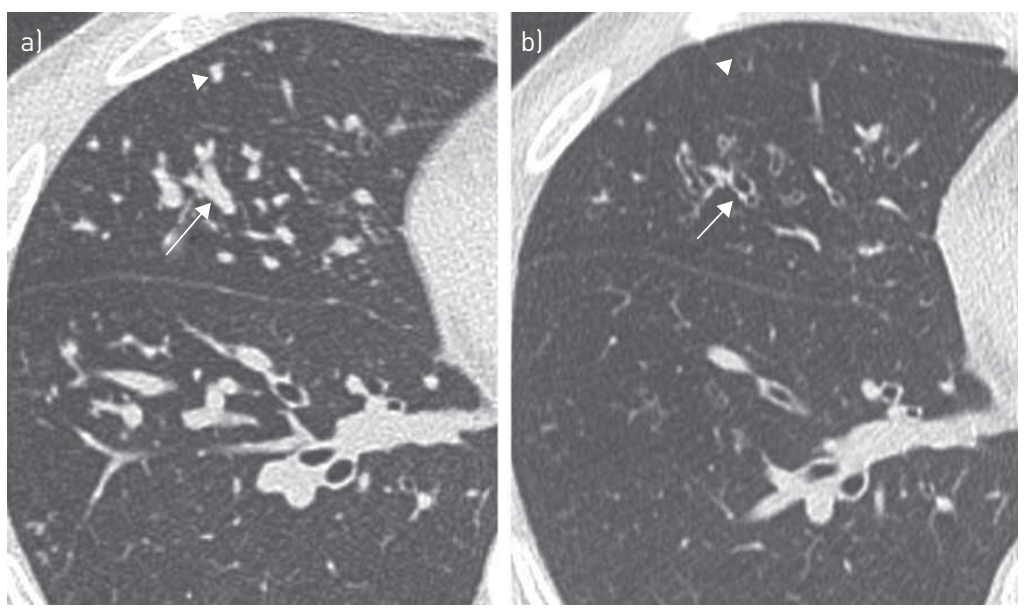

FIGURE 1 a) Pre-therapeutic and b) long-term (48 month) follow-up computed tomography examinations demonstrating dramatic improvement in mucus plugging under treatment with ivacaftor. Improvement in mucus plugging unmasks central (arrow) and peripheral (arrowhead) bronchiectasis leading to an increase in the bronchiectasis score of the middle lobe. 
ivacaftor treatment. Despite being only moderate, the correlation between interscan changes of FEV 1 and CF-CT score in our study was higher than that previously reported between changes of FEV $1 /$ forced vital capacity and visual score ( $\mathrm{r}=-0.51$ versus -0.39$)$ [14].

Our study has several limitations. First, due to the retrospective design, CT examinations were performed at different time points and only eight patients had both short- and long-term follow-up CT examinations and there was no standardisation of the CT parameters among the centres. However, only three of the 52 CT scans had slice thicknesses $>1.25 \mathrm{~mm}$. The remaining consisted in high-resolution protocols. Second, we did not evaluate interobserver agreement for visual scoring, since only one radiologist could receive intensive training in a reference centre. The limited number of available trained scorers is a well-known limitation of visual CT scores [15]. Lastly, evaluating treatment efficacy with CT raises concerns about radiation dose, even though recent improvements of the CT technology allow for dramatic dose reduction.

In conclusion, our study shows that CT is a valuable method for monitoring CF patients treated with ivacaftor. Decrease of mucus plugging and peribronchial thickening occurred during the first year and remained stable on long-term follow-up, whereas bronchiectasis score slightly increased, possibly because of improved visualisation, after mucus plugging clearance.

In CF patients treated with ivacaftor, improvement of $\mathrm{CT}$ abnormalities remains stable on longterm follow-up http://ow.ly/Zxu2B

Guillaume Chassagnon $^{1,2}$, Dominique Hubert ${ }^{2,3}$, Isabelle Fajac ${ }^{2,4}$, Pierre-Régis Burgel ${ }^{2,3}$ and Marie-Pierre Revel ${ }^{1,2}$ on behalf of the investigators

${ }^{1}$ Radiology Dept, Cochin Hospital, AP-HP, Paris, France. ${ }^{2}$ Université Paris Descartes, Sorbonne Paris Cité, Paris, France.

${ }^{3}$ Pulmonary Dept and Adult CF Centre, Cochin Hospital, AP-HP, Paris, France. ${ }^{4}$ Physiology Dept, Cochin Hospital, AP-HP, Paris, France.

Correspondence: Marie-Pierre Revel, Radiology Dept, Cochin Hospital, AP-HP, 27 Rue du Faubourg Saint-Jacques, 75014 Paris, France. E-mail: marie-pierre.revel@aphp.fr

Received: Nov 172015 | Accepted after revision: March 12 2016 | First published online: May 262016

Conflict of interest: Disclosures can be found alongside the online version of this article at erj.ersjournals.com

Acknowledgements: The investigators are: Isabelle Danner-Boucher (Pulmonary Dept, G\&R Laënnec Hospital, Nantes, France), Benoît Desrues (Pulmonary Dept, Pontchaillou Hospital, Rennes, France), Stéphane Dominique (Pulmonary Dept, Charles Nicolle Hospital, Rouen, France), Isabelle Durieu (Dept of Internal Medicine, Hospices Civils de Lyon et Université Claude Bernard Lyon1, Pierre-Bénite, France), Dominique Grenet (Pulmonary Dept, Foch Hospital, Suresnes, France), Anne-Cécile Henriet (Pulmonary Dept, Bretonneau Hospital, Tours, France), Anne Prevotat (Pulmonary Dept, Calmette Hospital, Lille, France).

\section{References}

1 Van Goor F, Hadida S, Grootenhuis PDJ, et al. Rescue of CF airway epithelial cell function in vitro by a CFTR potentiator, VX-770. Proc Natl Acad Sci USA 2009; 106: 18825-18830.

2 Accurso FJ, Rowe SM, Clancy JP, et al. Effect of VX-770 in persons with cystic fibrosis and the G551D-CFTR mutation. N Engl J Med 2010; 363: 1991-2003.

3 Ramsey BW, Davies J, McElvaney NG, et al. A CFTR potentiator in patients with cystic fibrosis and the G551D mutation. N Engl J Med 2011; 365: 1663-1672.

4 Davies JC, Wainwright CE, Canny GJ, et al. Efficacy and safety of ivacaftor in patients aged 6 to 11 years with cystic fibrosis with a G551D mutation. Am J Respir Crit Care Med 2013; 187: 1219-1225.

5 De Boeck K, Munck A, Walker S, et al. Efficacy and safety of ivacaftor in patients with cystic fibrosis and a non-G551D gating mutation. J Cyst Fibros 2014; 13: 674-680.

6 Hoare S, McEvoy S, McCarthy CJ, et al. Ivacaftor imaging response in cystic fibrosis. Am J Respir Crit Care Med 2014; 189: 484.

7 Hayes D, Long FR, McCoy KS, et al. Improvement in bronchiectasis on CT imaging in a pediatric patient with cystic fibrosis on ivacaftor therapy. Respiration 2014; 88: 345.

8 Sheikh SI, Long FR, McCoy KS, et al. Computed tomography correlates with improvement with ivacaftor in cystic fibrosis patients with G551D mutation. J Cyst Fibros 2015; 14: 84-89.

9 Wainwright CE, Vidmar S, Armstrong DS, et al. Effect of bronchoalveolar lavage-directed therapy on Pseudomonas aeruginosa infection and structural lung injury in children with cystic fibrosis: a randomized trial. JAMA 2011; 306: 163-171.

10 Miller MR, Hankinson J, Brusasco V, et al. Standardisation of spirometry. Eur Respir J 2005; 26: 319-338.

11 Horsley AR, Davies JC, Gray RD, et al. Changes in physiological, functional and structural markers of cystic fibrosis lung disease with treatment of a pulmonary exacerbation. Thorax 2013; 68: 532-539.

12 Whitwell F. A study of the pathology and pathogenesis of bronchiectasis. Thorax 1952; 7: 213-239.

13 McKone EF, Borowitz D, Drevinek P, et al. Long-term safety and efficacy of ivacaftor in patients with cystic fibrosis who have the Gly551Asp-CFTR mutation: a phase 3, open-label extension study (PERSIST). Lancet Respir Med 2014; 2: 902-910. 


\title{
Insights into ventilation-gas exchange coupling in chronic thromboembolic pulmonary hypertension
}

\author{
To the Editor:
}

Exercise intolerance due to excessive ventilation and dyspnoea are fundamental clinical features of patients with pulmonary vascular diseases [1]. Among these diseases, chronic thromboembolic pulmonary hypertension (CTEPH) is associated with the largest increase in exercise ventilation [2]. Understanding the mechanism(s) underlying excess exercise ventilation in CTEPH is clinically relevant when designing evidence-based therapeutic and rehabilitative strategies to improve patients' symptoms and quality of life.

Excess exercise ventilation in CTEPH has been traditionally ascribed to increased "wasted" ventilation, i.e. hypoperfusion of well-ventilated alveoli. In fact, the physiological dead space fraction of tidal volume (VD/VT ratio) at peak exercise not only predicts CTEPH after pulmonary embolism [3] but also improves after clinical [4] and surgical [5] treatment. As the end-tidal carbon dioxide tension $\left(P \mathrm{ETCO}_{2}\right)$ diminishes when ventilation is excessive relative to perfusion [6], increased $V \mathrm{D} / V \mathrm{~T}$ has been mechanistically linked to inordinately low $\mathrm{PETCO}_{2}$ in CTEPH [7]. A $\mathrm{PETCO}_{2}$ lower than arterial (a) carbon dioxide tension $\left(\mathrm{PCO}_{2}\right)$ (i.e. a positive $P(\mathrm{a}-\mathrm{ET}) \mathrm{CO}_{2}$ gradient) [6] also suggests impaired pulmonary perfusion as a potential explanation for low exercise $\mathrm{PETCO}_{2}$ in these patients [8].

Surprisingly, however, seminal studies comparing lung absorption of multiple inert gases showed no or a limited increase in $V \mathrm{D} / V \mathrm{~T}$ despite marked pulmonary arterial obstruction [9]. This prompts an alternative (or complementary) explanation for a low exercise $\mathrm{PETCO}_{2}$ in $\mathrm{CTEPH}$ : reduced alveolar $\mathrm{PCO}_{2}$ (hyperventilation) [10]. In fact, heightened neural drive has been found in pulmonary vascular diseases secondary to increased chemosensitivity [11] and/or higher afferent stimuli from "central" baro- and mechano-receptors [12]. Thus, alveolar hyperventilation under the stress of exercise may also explain the relatively lower $\mathrm{PETCO}_{2}$ in CTEPH. A hyperbolic correlation between increasing minute ventilation $\left(V^{\prime} \mathrm{E}\right) /$ carbon dioxide production $\left(V^{\prime} \mathrm{CO}_{2}\right)$ and decreasing arterial carbon dioxide tension $\left(\mathrm{PaCO}_{2}\right)$ in $\mathrm{CTEPH}$ is also suggestive of heightened neural drive and consistent with the alveolar equation [13].

There is another intriguing feature of the $\mathrm{PETCO}_{2}$ response that brings additional uncertainty about the meaning of a low exercise $\mathrm{PETCO}_{2}$ in CTEPH. Similar to pulmonary arterial hypertension (PAH) [9], $\mathrm{PETCO}_{2}$ paradoxically increases (instead of further decreasing as in normal subjects) as soon as CTEPH patients enter the recovery phase. In this context, evaluation of $\mathrm{PaCO}_{2}$ (or arterialised capillary $\left(P_{\mathrm{cCO}}\right)$ as its surrogate) [8] and $P(\mathrm{c}-\mathrm{ET}) \mathrm{CO}_{2}$ across the exercise-to-recovery transition might shed light on the mechanisms underlying the $\mathrm{PETCO}_{2}$ behaviour during recovery in $\mathrm{CTEPH}$. Thus, we reasoned that during recovery: 1) if $\mathrm{PCCO}_{2}$ remains stable or further decreases despite higher $P \mathrm{ETCO}_{2}$, a narrower $P(\mathrm{c}-\mathrm{ET}) \mathrm{CO}_{2}$ would suggest improved $V \mathrm{D} / V \mathrm{~T}$ (scenario 1); 2) conversely, if $\mathrm{PCCO}_{2}$ and $\mathrm{PETCO}_{2}$ increase proportionally leading to stable $P(\mathrm{c}-\mathrm{ET}) \mathrm{CO}_{2}$, lower neural drive would explain higher $P \mathrm{ETCO}_{2}$ (scenario 2); 3) but if a narrower $P(\mathrm{c}-\mathrm{ET}) \mathrm{CO}_{2}$ develops as a consequence of $P_{\mathrm{CCO}_{2}}$, increasing less than $P \mathrm{ETCO}_{2}$, improved $V \mathrm{D} / V \mathrm{~T}$ plus lower neural drive would explain a higher $\mathrm{PETCO}_{2}$ (scenario 3).

We therefore measured $\mathrm{P}_{\mathrm{CCO}_{2}}$ (arterialised ear lobe blood), $P(\mathrm{c}-\mathrm{ET}) \mathrm{CO}_{2}$ and plasma lactate 1 min before peak incremental exercise, at peak and in recovery at every minute up to the fifth minute of unloaded cycling. Responses from 10 patients (age 54-78 years, five males, mean \pm SE pulmonary arterial pressure $51.5 \pm 9.7 \mathrm{mmHg}$, pulmonary wedge pressure $9.2 \pm 3.4 \mathrm{mmHg}$, stable segmental perfusion defects after 6 months of anticoagulation) were contrasted with those from eight age- and sex-matched healthy subjects. As expected, patients had a lower peak oxygen uptake, $V^{\prime} \mathrm{CO}_{2}$ and arterial oxygen saturation measured by pulse oximetry $\left(S_{\mathrm{pO}_{2}}\right)(\mathrm{p}<0.05)$. Lactate corrected for peak work rate was higher in patients 